\title{
Squamous Metaplasia in Pleomorphic Adenoma: A Diagnostic and Prognostic Enigma
}

Swati Sharma $\cdot$ Monica Mehendiratta Nivedita Chaudhary • Vineet Gupta Maulshree Kohli · Anjana Arora ${ }^{1}$

Departments of Oral and Maxillofacial Pathology and Microbiology and ${ }^{1}$ Oral Medicine and Radiology, I.T.S. Dental College, Hospital and Research Centre, Greater Noida, India

Received: April 27, 2018

Revised: June 20, 2018

Accepted: July 14, 2018

Corresponding Author

Swati Sharma, MDS

Department of Oral and Maxillofacial Pathology and

Microbiology, I.T.S. Dental College, Hospital and

Research Centre, Greater Noida, Uttar Pradesh

201308, India

Tel: +91-0120-2331089

Fax: +91-0120-2323702

E-mail: sharmaswatisharma86@gmail.com
Pleomorphic adenoma (PA) is the most common benign salivary gland tumor. Histologically, squamous metaplasia has been reported in PA, but has rarely been documented as being extensive enough to cause significant misdiagnosis. Here, we present an unusual case of PA in a 50-yearold female patient presenting with swelling on the postero-lateral aspect of the palate for a week. Histopathologically, the tumor exhibited the features of conventional PA with extensive squamous metaplasia and giant keratotic lamellae in cyst-like areas. Such exuberant squamous metaplasia and keratin can be a diagnostic and prognostic pitfall and lead to overtreatment of the patient.

Key Words: Squamous metaplasia; Pleomorphic adenoma; Pathology
Pleomorphic adenoma (PA) is the most common benign salivary gland tumor, accounting for $54 \%-76 \%$ of all salivary neoplasia. The parotid gland is the most common site of PA. ${ }^{1}$ Approximately $8 \%$ of PA involve the minor salivary glands, whereas the palate is the most common site, accounting for $60 \%-65 \%$ of cases. Such tumors have been referred to by a great variety of names over the years (e.g., mixed tumors, enclavoma, branchioma, endothelioma, and enchondroma). The term "pleomorphic adenoma" suggested by Willis closely characterizes the unusual and varied histologic pattern of the lesion. PA demonstrates consistent cytogenetic abnormalities, chiefly involving the chromosome region 12q1315. The putative PA gene is PLAG 1 and has been mapped to chromosome 8q 12. Being pleomorphic, it exhibits the ability to differentiate into epithelial (ductal and nonductal) cells and mesenchyme-like tissue (chondroid, myxoid and osseous). ${ }^{2}$ Thus, it is composed of a mixture of glandular epithelium and myoepithelial cells within a mesenchyme-like tissue, and the proportion of each component varies widely among individual tumors. ${ }^{1}$ The histomorphological variations are so extensive that in an incisional biopsy specimen, diagnosis can be challenging. The variations in epithelial and mesenchyme-like components with or without dysplasia add to this dilemma. The present case-study dealt with a massive PA of the palate with a misleading history of a week and an extensive squamous metaplasia with giant keratotic lamellae in cyst-like areas. This extensive squamous metaplasia and keratin brought this tumor's diagnosis close to intracapsular (in situ) PA, muco-epidermoid carcinoma, adenoid or adeno-squamous cell carcinoma, conventional squamous cell carcinoma (SCC), carcinoma ex PA, and necrotizing sialometaplasia. The patient granted consent and institutional ethical clearance was given for this case report (ITSCDSR/L/2018/086).

\section{CASE REPORT}

A 50-year-old female patient reported with a chief complaint of swelling on the right postero-lateral aspect of the palate for the past 1 week. Intraorally, a swelling associated with central ulceration and a fibrino-purulent membrane covering was present on the right side of the hard palate, with an approximate size of 5.0 $\times 5.0 \times 1.5 \mathrm{~cm}^{3}$ (Fig. 1). The swelling was firm in consistency, 
non-tender, slightly movable and had an erythematous oedematous periphery. There were no associated palpable lymph nodes.

Further investigations were carried out, including hematological examination, computed tomography, aspiration cytology and biopsy. Hematological examination included complete blood count, prothrombin time, erythrocyte sedimentation rate, and routine blood sugar without any significant alarming result. Aspiration of the swelling did not yield much and was not helpful for evaluating the diagnosis. A subsequent cone beam computed tomography (CBCT) scan captured coronal sectional images revealing a well-defined dome-shaped soft tissue shadow measuring $2.19 \times 2.42 \mathrm{~cm}^{2}$, extending between the palatal aspect of 17 and 18, and mid-line of the palate medio-laterally. There was no evidence of bone resorption. The three dimensional image revealed an ill-defined posterior-most extent of this soft tissue swelling up to the oropharynx. No radiographic signs of malignancy such as invasive borders, irregular cortical boundary and aggressive bone destruction, root resorption, tooth displacement or periosteal reaction were evident (Fig. 2).

Wide local excision was conducted and the specimen was removed in toto in an uneventful surgery. Grossly, the tumor appeared well-encapsulated (Fig. 1), grayish-white, firm in consistency, smooth in texture and measured $2.6 \times 2.9 \times 1.7 \mathrm{~cm}^{3}$. A provisional diagnosis of lymphadenopathy and palatal minor salivary gland tumor were made.

Hematoxylin and eosin-stained sections revealed a well-delin-
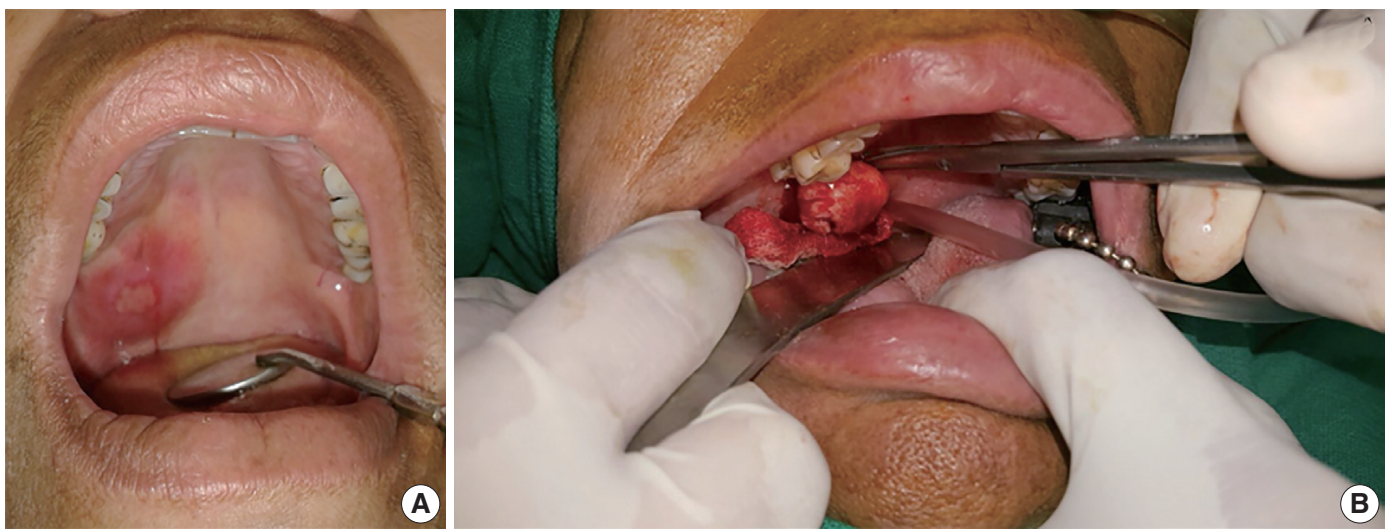

Fig. 1. (A) Swelling associated with central ulceration covered by a fibrino-purulent membrane and surrounded by palatal erythema can be seen on the right distal side of the hard palate, laterally. (B) A well-encapsulated tumor removed in toto from the palate.
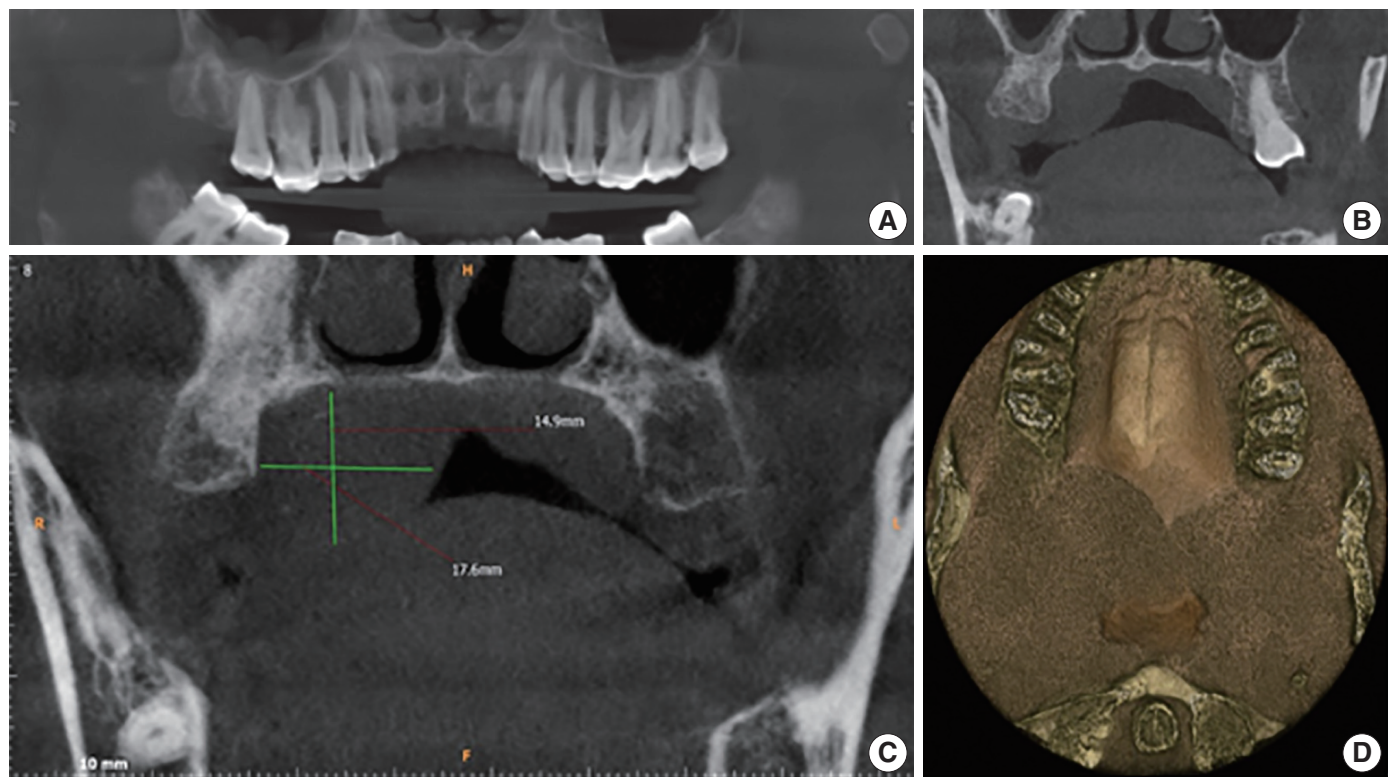

Fig. 2. (A) Reconstructed panoramic image shows the polyp at the level of the mid right maxillary sinus. (B) Axial section shows inflamed sinus lining involving the right maxillary sinus. (C) Cone beam computed tomography (CBCT) mid-coronal section of the right maxillary third molar (18) showing the soft tissue shadow palatally. (D) Two-three dimensional CBCT image showing the antero-posterior extent of the swelling. 
eated but partly encapsulated tumor mass flanked with abundant adipocytes. Under low magnification, the central pathology consisted of variably shaped, abundant ducts and massive squamous epithelium-lined cysts with extensive keratin in a myxoid and hyalinized stroma (Fig. 3). Under higher magnification, a classical bilayered ductal pattern with luminal cuboidal cells and variably-shaped, abluminal, myoepithelial cells was ascertained. The myoepithelial cell layers varied from a single layer to collar to an extensive collection, imparting the appearance of a swarm of bees. The other important feature of the lesion was florid squamous cells ( $50 \%$ of the tumor) arranged in nests, islands or in sheets with or without extensive cystic cavities containing massive keratotic lamellae and/or areas of degeneration. The cystic cavities were lined by $4-5$ compressed layers of hyperchromatic, stratified squamous epithelium with hypergranulosis and sparse mitotic figures in the outer cells, but no evidence of atypia. The lining epithelium showed bud-shaped projections in the stromal tissue away from the cystic cavities (Fig. 3).

The stroma also revealed histogenetic diversity with extensive myxoid, fibrous to hyalinized areas with evidence of dystrophic calcification. The features were clearly suggestive of PA with florid squamous metaplasia and keratin-filled cysts. Post-operative healing of the patient was uneventful without any recurrence till 1.5 years after excision.

The presence of an extensive squamous component with keratin lamellae in the tumor background with ducts and variably shaped epithelial cells dispersed in a myxoid and hyalinized background created a multitude of differential diagnoses. The absence of frank mucous and intermediate cells as well as extensive mucin pooling helped distinguish from mucoepidermoid carcinoma. Conventional SCC was considered, as it may invade or entrap nontumorous salivary glands, but was ultimately ruled out due to the presence of extensive morphological, epithelial and stromal diversity with no evidence of dysplasia. Adenoid SCC and adenosquamous cell carcinoma owing to the presence of squamous and glandular components were also considered as differentials, although the former malignancy does not show any true glands/ ducts or intracytoplasmic mucins. The latter and other malignancies such as in-situ (intracapsular) PA and carcinoma ex-PA were ruled out for several reasons. First, the received tissue was in toto
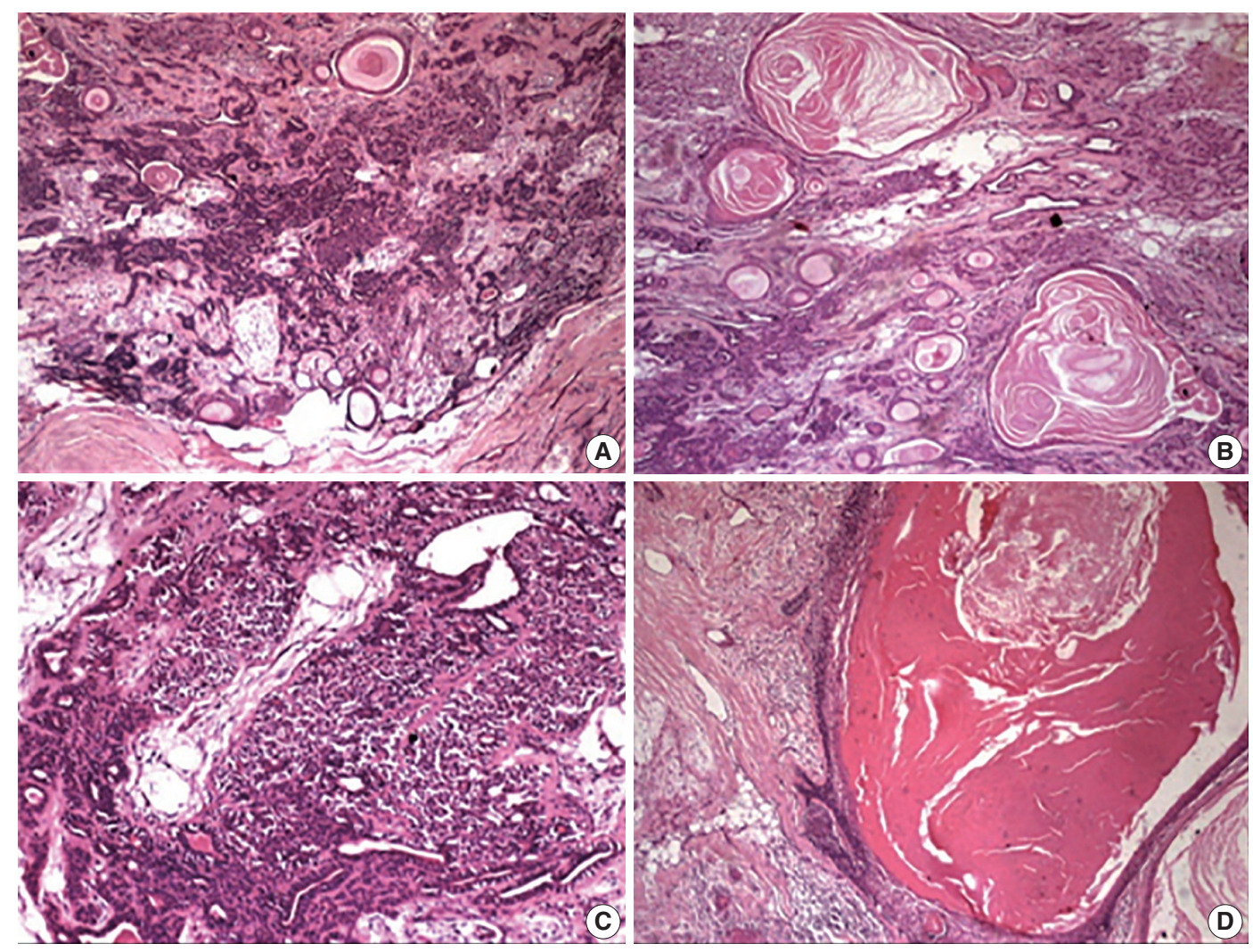

Fig. 3. (A) The periphery of the tumor with classical features of pleomorphic adenoma having abundant ducts dispersed in a myxoid and hyalinized background with multiple keratin-filled cystic areas. (B) Multitude of features, including variably shaped epithelial cells, ducts, and squames. Keratin-filled cysts dispersed in the myxoid stroma. (C) Abundant myoepithelial cells around the ducts giving the appearance of a swarm of bees. (D) Massive epithelial lined cysts with keratotic lamellae and the lining budding into the stroma. 
and thoroughly examined for evidence of any atypical changes. Second, there was an absence of cellular and nuclear atypia, necrosis, capsular invasion, an aggressive growth pattern and nerve/surrounding tissue permeation.

Clinically, because the tumor was located on the palate with a short history of presentation, a diagnosis of necrotizing sialometaplasia or chronic sialadenitis was also considered, but ultimately ruled out due to the absence of necrosis and presence of rich morphological diversity consistent with the diagnosis of PA.

Another important feature of note in the present case was squamous metaplasia, which could have been the result of the fine needle aspiration cytology (FNAC) conducted prior to excision of the lesion. However, the exuberant amount of squamous metaplasia evident in the lesion did not appear to be correlated with a needle-induced change.

\section{DISCUSSION}

The present case is a classical case of PA without cytological atypia, but demonstrating extensive squamous metaplasia, which can be of serious concern. Squamous metaplasia is an incidental microscopic finding in various benign and malignant (de-novo or induced) tumors in humans or animal models. The origin is unknown and has been associated erratically with the intra-tumoral/ tissue environment, like trauma, infarction/ischemia and repair following infarction. ${ }^{3}$ Squamous metaplasia has been experimentally induced by arterial ligation in rat salivary glands by Dardick et al., ${ }^{4}$ and appears to have formed via the gradual dedifferentiation and hyperplasia of the acinar-intercalated duct system. Tonofilaments and desmosomes begin to appear in the luminal and abluminal myoepithelial cells, and thus keratinization of central cells materializes. ${ }^{4}$ The varying degree of squamous metaplasia could be a consequence of the rapidity and ease of switch in the genetic programming of cytokeratin filaments induced by ischemia in the salivary glands, and so the most probable etiology for this change appears to be ischemia. ${ }^{3}$ FNAC for diagnostic purposes has been shown to induce the same in tumors during histopathological evaluation. ${ }^{5}$ FNAC was conducted in our case, but there was no evidence of necrosis/repair in the sections, and the amount of squamous metaplasia appeared to be correlated with the extent of injury induced by ischemia.

Foci of squamous cells are an integral feature of PA, but extensive squamous metaplasia is uncommon and can be easily misinterpreted as SCC, especially in FNAC and incisional biopsies due to the limited and selective sampling. In addition, diagnosis becomes challenging in the absence of chondro-myxoid stroma, making it imperative to understand this diagnostic pitfall. ${ }^{6}$

The presence of squamous metaplasia is also a prognostic pitfall, as its transition into SCC has been further emphasized by Takegawa $e t$ al. ${ }^{7}$ in the submandibular glands of rats by the application of potassium iodide. Takegawa et al. observed the development of squamous metaplasia in proliferative ductules and interlobular ducts that apparently transited to SCC, and emphasized that this occurred via a non-genotoxic, proliferation-dependent mechanism. ${ }^{7}$

To summarize, similar case reports have been provided by various authors with or without application of immunohistochemistry (IHC) markers. One case described a 32-year-old patient with $45 \%$ of the tumor consisting of squamous cells, wherein IHC helped distinguish the squamous metaplastic cells from SCC. ${ }^{8}$ The presence of low molecular weight cytokeratin and $\mathrm{p} 63$ in squamous cells helps rule out SCC or even reactive squamous hyperplasia in such PA cases. ${ }^{9}$ Multiple IHC markers are used to ascertain differences between glandular cells or metaplasticallyformed squamous cells. Although no conclusive differences have been established using cytokeratin or even MIB- $1^{8}$ (a proliferative marker), Ki-67 as used by Goulart et al..$^{10}$ had a higher proliferative index in the epithelial lining of a large keratin cyst.

Diagnosis of PA requires physical examination, CBCT, cytology and histopathology. FNAC and incisional biopsy can help determine the proper management regimen, but must be thoroughly sampled to rule out any misdiagnosis, especially in cases of misleading short histories like our present case. Other supportive investigations like computed tomography scanning and magnetic resonance imaging can provide information on the location and size of the tumor and its extension into surrounding superficial and deep structures.

The treatment for PA is surgical excision, and although radiotherapy is not indicated, correct diagnosis is essential to avoid overtreatment. ${ }^{2}$ The present case, to the best of our knowledge, is among the first 20 cases reported in the English language literature and thus a rarity. The misleading short history of one week, enormous size of $5.0 \times 5.0 \times 1.5 \mathrm{~cm}^{3}$ and massive squamous islands could have led to an incorrect diagnosis of malignancy. Thus, the thorough examination of samples, particularly in FNAC and incisional cases, is important.

\section{ORCID}

Swati Sharma: https://orcid.org/0000-0003-1124-568X

Monica Mehendiratta: https://orcid.org/0000-0001-6841-8591

Nivedita Chaudhary: https://orcid.org/0000-0001-9134-8835

Vineet Gupta: https://orcid.org/0000-0002-2333-1625 


\section{Conflicts of Interest}

No potential conflict of interest relevant to this article was reported.

\section{REFERENCES}

1. Neville B, Damm DD, Allen C, Bouquot J. Oral and maxillofacial pathology. 3rd ed. St. Louis: Elsevier; 2009; 473-506.

2. Rajendran R, Sivapathasundharam B. Shafer's text book of oral pathology. 7th ed. Noida: Elsevier, 2012; 223-57.

3. Kaveri H, Gopalkrishnana K, Venkatesh A. Cystic and florid squamous metaplasia in pleomorphic adenoma of palate: a diagnostic dilemma. Asian J Med Sci 2014; 5: 108-10.

4. Dardick I, Jeans MT, Sinnott NM, Wittkuhn JF, Kahn HJ, Baumal R. Salivary gland components involved in the formation of squamous metaplasia. Am J Pathol 1985; 119: 33-43.

5. Jaishankar HP, Hegde U, Nagpal B. Florid squamous metaplasia and keratin cyst formation in palatal minor salivary gland tumor: a diagnostic challenge. Int J Health Sci Res 2016; 6: 516-20.

6. Compagno J, Wong RT. Intranasal mixed tumors (pleomorphic adenomas): a clinicopathologic study of 40 cases. Am J Clin Pathol 1977; 68: 213-8.

7. Takegawa K, Mitsumori K, Onodera H, et al. Induction of squamous cell carcinomas in the salivary glands of rats by potassium iodide. Jpn J Cancer Res 1998; 89: 105-9.

8. Lam KY, Ng IO, Chan GS. Palatal pleomorphic adenoma with florid squamous metaplasia: a potential diagnostic pitfall. J Oral Pathol Med 1998; 27: 407-10.

9. Lim S, Cho I, Park JH, Lim SC. Pleomorphic adenoma with exuberant squamous metaplasia and keratin cysts mimicking squamous cell carcinoma in minor salivary gland. Open J Pathol 2013; 3: 113-6.

10. Goulart MC, Freitas-Faria P, Goulart GR, et al. Pleomorphic adenoma with extensive squamous metaplasia and keratin cyst formations in minor salivary gland: a case report. J Appl Oral Sci 2011; 19: 182-8. 